\section{Aderência a medidas de proteção individual contra a hepatite B entre cirurgiões-dentistas e auxiliares de consultório dentário}

\section{Adherence to personal protection against hepatitis $B$ among dentists and dental assistants}

\section{Leila Posenato Garcia}

Vera Lúcia Guimarães Blank

\section{Nelson Blank}

Programa de Pós-Graduação em Saúde Pública, Centro de Ciências da Saúde, Universidade Federal de Santa Catarina

\section{Resumo}

Objetivo: O vírus da hepatite B é um risco ocupacional reconhecido na odontologia. Além de aderir às precauções padrão, todos os profissionais da odontologia devem se proteger através da vacinação contra a hepatite B e confirmação da imunidade. Este estudo verificou a aderência às medidas de proteção individual contra a hepatite $B$, incluindo a vacinação e o uso de equipamentos de proteção individual, entre cirurgiões-dentistas e auxiliares de consultório dentário do município de Florianópolis, localizado no sul do Brasil. Métodos: Neste estudo transversal, os dados foram coletados através de questionários autoaplicáveis. Foram obtidas respostas de 289 cirurgiões-dentistas e 104 auxiliares. Resultados: A prevalência da vacinação completa contra a hepatite B foi $73,4 \%$ entre os dentistas e $39,4 \%$ entre os auxiliares. Apenas $32,1 \%$ e $21,9 \%$ deles, respectivamente, monitoraram a resposta vacinal. A vacinação completa foi positivamente associada com o ano de formatura a partir de 1997 (RP = 1,16; IC $^{95 \%}=1,01-1,33$ ) e atuação predominantemente em especialidade cirúrgica $\left(\mathrm{RP}=1,24 ; \mathrm{IC}^{95 \%}=1,02-1,51\right)$ entre os dentistas e com a realização de curso de formação entre os auxiliares $\left(\mathrm{RP}=1,96\right.$; $\mathrm{IC}^{95 \%}=$ 1,23-3,14). O uso constante de luvas, máscara e óculos de proteção foi mais elevado entre os dentistas do que entre os auxiliares. Conclusão: São indicadas campanhas visando a vacinação daqueles que não o fizeram ou não completaram o esquema vacinal e informações sobre a necessidade do monitoramento da resposta vacinal. Medidas educativas são recomendadas para elevar a aderência às medidas de proteção pessoal, voltadas especialmente aos auxiliares.

Palavras-chave: Vacinas contra hepatite B. Prevalência. Hepatite B, prevenção \& controle. Recursos Humanos em Odontologia. Saúde do Trabalhador. 
Abstract

Objective: The Hepatitis B virus poses a recognized occupational risk to dental care workers. All dental care workers must be protected through immunization, with completion of vaccine series, and monitoring of vaccine response. This study examined the adherence to personal protection against hepatitis $B$, including vaccination and use of personal protective equipment, among dentists and dental assistants in the city of Florianopolis, South of Brazil. Methods: In this cross-sectional study, data were collected through self-reported questionnaires. Responses were obtained from 289 dentists and 104 dental assistants. Results: The prevalence of complete hepatitis B series vaccination was $73.4 \%$ among dentists and $39.4 \%$ among dental assistants. Only $32.1 \%$ and $21.9 \%$ of them, respectively, monitored vaccine response. A complete vaccine series was associated with year of graduation as of $1997(\mathrm{PR}=$ 1.16; IC $^{95 \%}=1.01-1.33$ ) and working mainly in a surgical specialty $\left(\mathrm{PR}=1.24\right.$; $\mathrm{IC}^{95 \%}=$ 1.02-1.51) among dentists, and with attending a training program among assistants $\left(\mathrm{PR}=1.96 ; \mathrm{IC}^{95 \%}=1.23-3.14\right)$. The proportion of always wearing gloves, masks, and protective eyewear was higher among dentists than assistants. Conclusion: Campaigns are necessary to vaccinate those who neither have been vaccinated nor have completed vaccine series and to inform about the need of vaccine response monitoring. Educational training is recommended to improve adherence to personal protection, specially focused on dental assistants.

Keywords: Hepatitis B vaccines. Prevalence. Hepatitis B, Prevention \& Control. Dental Staff. Occupational Health.

\section{Introdução}

A hepatite B apresenta distribuição universal. Estima-se que aproximadamente dois bilhões de pessoas, um terço da população mundial, já tiveram contato com o vírus da hepatite B (HBV) e 325 milhões tornaram-se portadores crônicos. No Brasil, a doença apresenta endemicidade variável. Endemicidades baixas (até $2 \%$ ) e intermediárias (2\% a 7\%) são predominantes; entretanto, algumas regiões como sudeste do Pará, oeste de Santa Catarina e oeste do Paraná apresentam alta endemicidade (superior a $7 \%)^{1}$.

O HBV é um risco ocupacional reconhecido para os profissionais da odontologia. Exposições percutâneas ou de mucosas ao sangue de indivíduos infectados pelo HBV representam a principal fonte de transmissão ocupacional, já que quantidades diminutas de sangue são suficientes para transmitir a infecção. O HBV também está presente em outros fluidos corporais, incluindo a saliva e o fluido crevicular, que também podem ser transmissores ${ }^{2,3}$. A transmissão através de respingo de sangue à mucosa ocular e mordedura já foi documentada. Além disso, a elevada resistência ambiental do HBV - que sobrevive mais de uma semana no sangue seco em temperatura ambiente - associada ao fato de que muitos profissionais da saúde infectados pelo HBV não recordam ter sofrido exposição a sangue contaminado, leva a crer que muitas infecções ocupacionais resultam da inoculação do HBV em lesões cutâneas (como arranhões, abrasões, queimaduras) ou em mucosas ${ }^{3}$.

Após lesões provocadas por agulhas contaminadas com sangue contendo HBV, a probabilidade de desenvolver evidência sorológica de infecção pelo HBV está altamente relacionada ao estado do pacientefonte. A probabilidade de desenvolver hepatite B clínica é de $22 \%-31 \%$ e a probabilidade de desenvolver evidência sorológica de infecção pelo HBV é de 37\%-62\% quando o sangue é positivo para os antígenos HBs (HBs-Ag) e Hbe (HBe-Ag). Quando o 
sangue é HBs-Ag positivo e Hbe-Ag negativo, a probabilidade de desenvolver hepatite B clínica é de 1\%-6\% e a probabilidade de desenvolver evidência sorológica da infecção pelo HBV é de 23\%-37\%. O HBV é mais facilmente transmitido após acidente envolvendo fluidos corporais e apresenta maior grau de infectividade do que o vírus da hepatite $\mathrm{C}$ (HCV) e o vírus da imunodeficiência humana (HIV) ${ }^{3}$.

Medidas de proteção individual são indicadas para evitar a transmissão ocupacional do HBV na odontologia, incluindo o uso de equipamentos de proteção individual (luvas, máscara, óculos de proteção e jaleco) e a vacinação de todo o pessoal que realiza tarefas que envolvam contato com sangue, outros fluidos corporais, instrumentos perfurocortantes ou superfícies contaminadas por fluidos corporais. A vacinação deve ser completada preferencialmente ainda durante o treinamento, antes que os indivíduos tenham contato com as substâncias mencionadas ${ }^{3}$.

No Brasil, a vacinação contra a hepatite $\mathrm{B}$ é recomendada universalmente para recém-nascidos, adolescentes até 19 anos $^{1}$ e pessoas com risco acrescido para adquirir a infecção, entre elas os profissionais da odontologia. Devido às características da prática odontológica envolvendo trabalho com instrumentos perfurocortantes e fluidos corporais em um campo de visão restrito, a vacinação anti-HBV é uma medida prioritária dentre os procedimentos de controle de infecção². Em julho de 1995 foi iniciada a primeira campanha de vacinação contra a hepatite B para profissionais e estudantes da odontologia. Desde então, o Programa Nacional de Imunizações disponibiliza gratuitamente a vacina contra a hepatite B para essa população nos centros da rede básica de saúde.

A vacina é administrada em três doses (0, 1 e 6 meses), sendo a realização do esquema vacinal completo necessária para a imunização. Contudo, aproximadamente $10 \%$ a $20 \%$ dos indivíduos vacinados não alcançam os títulos protetores de anti$\operatorname{corpos}^{4}$. O Ministério da Saúde ${ }^{2}$ recomen- da que, 30 dias após a administração da última dose do esquema vacinal contra a hepatite B, os membros da equipe odontológica verifiquem se foram efetivamente imunizados através de exames imunológicos.

O presente estudo objetiva verificar a aderência ao uso dos equipamentos de proteção individual e estimar a prevalência da vacinação e confirmação da imunidade contra a hepatite $\mathrm{B}$ e a magnitude da associação entre a vacinação completa e variáveis demográficas, ocupacionais e educacionais entre cirurgiões-dentistas (CDs) e auxiliares de consultório dentário (ACDs) do município de Florianópolis, SC.

\section{Método}

Florianópolis é a capital do Estado de Santa Catarina, localizado na região sul do Brasil. A população deste estudo transversal constituiu-se de 1.272 CDs domiciliados no município de Florianópolis em maio de 2003 e dos ACDs que trabalhavam com esses CDs. O tamanho da amostra foi dimensionado para atender ao objetivo geral do estudo, que foi determinar a prevalência de exposições ocupacionais a material biológico no período de um ano ${ }^{5}$.

Os 326 CDs que fizeram parte da amostra foram selecionados através de amostragem probabilística sistemática a partir da listagem em ordem alfabética dos CDs registrados no Conselho Regional de Odontologia de Santa Catarina (CRO/SC). Considerando o fato de que nem todas as pessoas que trabalham como ACDs estão registradas no $\mathrm{CRO} / \mathrm{SC}$, não foi possível estimar o tamanho da amostra a partir do tamanho da população, que era desconhecido. Em função disso, a amostra de ACDs foi formada por aqueles que trabalhavam com os CDs selecionados. Além disso, alguns CDs poderiam relutar em permitir a participação de seu ACD em um estudo no qual não estariam participando, especialmente considerando que o estudo trata da saúde do trabalhador, pela qual eles também são responsáveis. 


\begin{tabular}{|ll|}
\hline $1^{\circ}$ nível & Variáveis demográficas: sexo, idade \\
\hline $2^{\circ}$ nível & Variáveis ocupacionais: ano de formatura, especialidade, docência \\
\hline $3^{\circ}$ nível & $\begin{array}{l}\text { Variáveis educacionais: pós-graduação, curso de atualização nos últimos dois } \\
\text { anos, curso envolvendo biossegurança nos últimos dois anos }\end{array}$ \\
\hline & Vacinação contra a hepatite B entre cirurgiões-dentistas \\
\hline
\end{tabular}

Figura 1 - Modelo hierárquico de análise.

Figure 1 - Hierarchic analysis model.

Os dados foram coletados entre maio e julho de 2004, através de questionário auto-aplicável padronizado, previamente testado com CDs e ACDs que não faziam parte da amostra. Os questionários não identificaram os profissionais que forneceram as informações, o que possibilitou a manutenção do anonimato dos participantes. O estudo foi realizado de acordo com os princípios éticos que constam na Resolução 196/1996 do Conselho Nacional de Saúde. A coleta dos dados foi iniciada apenas após o recebimento do parecer favorável fornecido pelo Comitê de Ética na Pesquisa com Seres Humanos (CEPSH) da Universidade Federal de Santa Catarina (projeto 043/2004).

As perguntas relacionadas à vacinação contra a hepatite B foram: "Você tomou a vacina contra a hepatite B?". Em caso afirmativo, "Quantas doses?" e "Se você tomou as três doses da vacina, realizou exame de sangue para verificar se adquiriu imunidade contra a hepatite B?".

A respeito da aderência às precauções padrão, verificou-se a freqüência de uso de todos os equipamentos de proteção individual (EPIs), incluindo luvas, máscara, jaleco, óculos e gorro.

Após a análise estatística descritiva das variáveis, foi realizada análise bivariável, utilizando o exato de Fisher para estimar diferenças entre as proporções. Na análise multivariável, utilizou-se regressão de Poisson para verificar a associação entre a realização do esquema vacinal completo entre CDs e variáveis demográficas e ocupacionais. A modelagem foi orientada pelo modelo hierárquico de análise ilus- trado na Figura 1, com regressão para trás, nível por nível. Foram mantidas no modelo as variáveis que apresentaram associação estatística com o desfecho com um valor $p \leq 0,20$, visando o controle de possíveis fatores de confusão. Foram calculados intervalos de confiança de $95 \%$ e consideradas estatisticamente significativas as associações com um valor $p \leq 0,05$. Os programas utilizados para as análises estatísticas foram Epi-Info 2002 e Stata versão 9.0.

\section{Resultados}

Foram obtidas as respostas de 289 CDs e 104 ACDs, representando uma proporção de não-resposta de aproximadamente $12,0 \%$. A prevalência de exposições ocupacionais a material biológico no último ano foi $39,1 \%$ entre os CDs e $39,4 \%$ entre os $\mathrm{ACDs}^{5}$.

A Tabela 1 apresenta o número de observações e freqüências nas categorias das variáveis estudadas entre CDs. A idade média dos CDs pesquisados foi 39,2 anos (25-73). Pouco mais da metade eram mulheres $(51,2 \%)$, sendo que proporção dos sexos na amostra foi similar à proporção na população de CDs. A maior titulação de $46 \%$ era graduação, de $39,1 \%$ especialização e 14,9\% tinham mestrado e/ou doutorado. Aproximadamente um décimo deles $(9,7 \%)$ exercia predominantemente uma especialidade cirúrgica (cirurgia, periodontia ou implantodontia) e 19,0\% exerciam atividade de docência. A maioria $(68,6 \%)$ havia realizado curso de atualização nos últimos dois anos, mas apenas $22,5 \%$ haviam realizado curso que envol- 
vesse biossegurança nos últimos dois anos.

O número de observações e freqüências nas categorias das variáveis estudadas entre ACDs estão apresentados na Tabela 2. Entre os ACDs pesquisados, a média de idade foi 28,4 anos (17-48). A maioria eram mulheres $(97,1 \%), 85,4 \%$ haviam completado o ensino médio, mas apenas $36,0 \%$ relataram ter realizado curso de formação de ACD.

A Tabela 3 apresenta a prevalência da realização do esquema vacinal completo contra a hepatite B (pelo menos três doses) e do uso constante de EPIs entre CDs e ACDs. A vacinação completa foi referida por $73,4 \%$ dos CDs e $39,4 \%$ dos ACDs. Desses, $32,1 \%$ dos CDs e $21,9 \%$ dos ACDs haviam realizado exame sorológico para monitoramento da resposta vacinal. Dentre os 77 sujeitos que realizaram teste para verificar a imunidade (68 CDs e 9 ACDs), 5 CDs $(6,5 \%)$ afirmaram ter recebido seis doses, indicando a repetição do esquema vacinal. Nenhum ACD indicou ter recebido mais de três doses da vacina. A prevalência do uso constante de luvas, óculos de proteção e máscara foi maior entre os CDs do que entre os ACDs. Quanto ao uso de jaleco e gorro, as prevalências foram semelhantes.

A análise bruta da associação entre vacinação completa contra a hepatite B e variáveis demográficas e ocupacionais entre ACDs está apresentada na Tabela 4. Entre as variáveis testadas, apenas a realização de curso de formação de ACD esteve estatisticamente associada a uma maior preva-

Tabela 1 - Número de observações e freqüências nas categorias das variáveis estudadas entre cirurgiões-dentistas ( $n=289$ ), Florianópolis, Brasil, 2004.

Table 1 - Number of observations and frequencies in the studied variables' categories among dentists $(n=289)$, Florianopolis, Brazil, 2004.

\begin{tabular}{|c|c|c|}
\hline Variáveis & $\mathrm{n}^{*}$ & $\%$ \\
\hline \multicolumn{3}{|l|}{ Sexo } \\
\hline Feminino & 148 & 51,2 \\
\hline Masculino & 141 & 48,8 \\
\hline \multicolumn{3}{|l|}{ Idade } \\
\hline$<40$ anos & 161 & 55,9 \\
\hline$\geq 40$ anos & 127 & 44,1 \\
\hline \multicolumn{3}{|l|}{ Ano da formatura } \\
\hline$<1997$ & 220 & 76,1 \\
\hline$\geq 1997$ & 69 & 23,9 \\
\hline \multicolumn{3}{|l|}{ Pós-Graduação } \\
\hline Não & 156 & 54,0 \\
\hline Sim & 133 & 46,0 \\
\hline \multicolumn{3}{|l|}{ Especialidade } \\
\hline Não-cirúrgica & 261 & 90,3 \\
\hline Cirurgia, periodontia, implantodontia & 28 & 9,7 \\
\hline \multicolumn{3}{|l|}{ Docência } \\
\hline Não & 234 & 81,0 \\
\hline Sim & 55 & 19,0 \\
\hline \multicolumn{3}{|l|}{ Curso de atualização nos últimos 2 anos } \\
\hline Não & 90 & 31,4 \\
\hline Sim & 197 & 68,6 \\
\hline \multicolumn{3}{|l|}{ Curso de biossegurança nos últimos 2 anos } \\
\hline Não & 224 & 77,5 \\
\hline Sim & 65 & 22,5 \\
\hline
\end{tabular}

* Número de observações válidas / * Number of valid observations 
Tabela 2 - Número de observações e freqüências nas categorias das variáveis estudadas entre auxiliares de consultório dentário $(n=104)$, Florianópolis, Brasil, 2004.

Table 2 - Number of observations and frequencies in the studied variables' categories among dental assistants $(n=104)$, Florianopolis, Brazil, 2004.

\begin{tabular}{lcc}
\hline Variáveis & $\mathrm{n}^{*}$ & $\%$ \\
\hline Sexo & 101 & \\
$\quad$ Feminino & 3 & 97,1 \\
$\quad$ Masculino & & 2,9 \\
Idade & 51 & 50,5 \\
$\quad<27$ anos & 50 & 49,5 \\
$\quad \geq 27$ anos & & \\
Escolaridade & 88 & 85,4 \\
$\quad$ ensino médio completo & 15 & 14,6 \\
$\quad<$ ensino médio completo & & \\
Tempo de trabalho & 69 & 66,3 \\
$\quad<7$ anos & 35 & 33,7 \\
$\quad \geq 7$ anos & & 64,0 \\
Curso de formação & 64 & 36,0 \\
$\quad$ Não & 36 & \\
$\quad$ Sim
\end{tabular}

* Número de observações válidas / * Number of valid observations

Tabela 3 - Prevalência da vacinação completa contra a hepatite B e do uso constante dos equipamentos de proteção individual (EPIs) entre cirurgiões-dentistas (CDs) e auxiliares de consultório dentário (ACDs), Florianópolis, Brasil, 2004.

Table 3 - Prevalence of complete vaccination series against hepatitis $B$ and always wearing personal protective equipment among dentists (CDs) and dental assistants (ACDs), Florianopolis, Brazil, 2004.

\begin{tabular}{lcccc}
\hline \multirow{2}{*}{ Variáveis } & \multicolumn{2}{c}{ Ocupações } & \multicolumn{2}{c}{ ACDs } \\
& $n$ & $\%$ & $n$ & $\%$ \\
\hline Vacinação completa contra a hepatite B & 212 & 73,4 & 41 & 39,4 \\
Sempre usa luvas & 263 & 91,0 & 81 & 77,9 \\
Sempre usa óculos de proteção & 172 & 59,5 & 23 & 22,1 \\
Sempre usa máscara & 271 & 93,8 & 47 & 45,2 \\
Sempre usa jaleco (qualquer tipo) & 268 & 92,7 & 95 & 91,3 \\
Sempre usa jaleco (mangas longas) & 139 & 48,1 & 42 & 40,4 \\
Sempre usa gorro & 81 & 28,0 & 27 & 26,0 \\
\hline
\end{tabular}

lência de vacinação ( $\mathrm{p}=0,006)$.

A Tabela 5 apresenta as análises bruta e ajustada, através de regressão de Poisson, da associação entre a vacinação completa contra a hepatite $\mathrm{B}$ e variáveis demográficas, ocupacionais e educacionais entre os CDs. Na análise bruta, nenhuma variável apresentou associação estatistica- mente significativa ( $\mathrm{p} \leq 0,05)$ com o desfecho. Contudo, após o ajuste, ano da formatura ( $\mathrm{p}=0,039)$ e especialidade ( $\mathrm{p}=$ $0,028)$ passaram a demonstrar associação. Os dentistas formados a partir de 1997 apresentaram uma chance de ter vacinação completa 16,0\% maior do que aqueles formados antes daquele ano. Os dentistas 
Tabela 4 - Análise bruta da associação entre vacinação completa contra a hepatite B e variáveis demográficas e ocupacionais entre auxiliares de consultório dentário, Florianópolis, Brasil, 2004. Table 4 - Crude analysis of the association among complete vaccination series against hepatitis $B$ and demographic and occupational variables among dental assistants, Florianopolis, Brazil, 2004.

\begin{tabular}{|c|c|c|c|c|}
\hline Variáveis & $\begin{array}{l}\text { Prevalência da } \\
\text { vacinação (\%) }\end{array}$ & $\begin{array}{c}\text { Razão de } \\
\text { prevalências }\end{array}$ & IC 95\%* & Valor $\mathrm{p}^{* *}$ \\
\hline Idade & & & & 0,543 \\
\hline$<27$ anos & 43,1 & 1,0 & - & \\
\hline$\geq 27$ anos & 36,0 & 0,83 & 0,$51 ; 1,36$ & \\
\hline Tempo de trabalho & & & & 0,206 \\
\hline$<7$ anos & 34,8 & 1,0 & - & \\
\hline$\geq 7$ anos & 48,6 & 1,39 & 0,$87 ; 2,23$ & \\
\hline Escolaridade & & & & 0,579 \\
\hline$<$ ensino médio completo & 38,6 & 1,0 & - & \\
\hline$\geq$ ensino médio completo & 46,7 & 1,21 & 0,$66 ; 2,20$ & \\
\hline Curso de formação & & & & 0,006 \\
\hline Não & 29,7 & 1,0 & - & \\
\hline Sim & 58,3 & 1,96 & 1,$23 ; 3,14$ & \\
\hline
\end{tabular}

* Intervalos de confiança de $95 \%$ / $* 95 \%$ confidence intervals

** Teste exato de Fisher bicaudal / ** Two-tailed Fisher's exact test

que exerciam predominantemente uma especialidade cirúrgica (cirurgia, periodontia ou implantodontia) apresentaram uma chance de ter vacinação completa $24,0 \%$ maior do que aqueles que exerciam predominantemente clínica geral ou outra especialidade.

\section{Discussão}

Os dados deste estudo foram coletados a partir de uma amostra de CDs e ACDs do município de Florianópolis e, portanto, podem não representar a realidade da população de CDs e ACDs brasileiros. A validade dos dados coletados através do uso de questionários auto-aplicáveis está sujeita a vieses de resposta e de memória. Tendo em vista a natureza da investigação, pode-se especular que os resultados superestimam a aderência às medidas de proteção individual, já que os sujeitos tendem a reportar comportamentos aceitáveis mesmo quando não os adotam. Contudo, os dados são capazes de fornecer um panorama sobre a aderência a medidas de proteção individual contra a hepatite $\mathrm{B}$ na população estudada.
Exposições ocupacionais a material biológico podem ocorrer através de lesões percutâneas (por exemplo, perfuração ou corte da pele íntegra) e do contato de sangue, tecidos ou fluidos corporais potencialmente infectantes com as mucosas ocular, nasal, bucal ou pele não íntegra. A maioria das exposições ocupacionais ao HBV na odontologia são preveníveis através da adoção das precauções padrão, que incluem o uso de luvas, máscara, gorro e jaleco ou avental ${ }^{3}$. O uso de luvas minimiza o potencial para o contato da pele não intacta dos trabalhadores da odontologia com sangue, saliva e mucosa dos pacientes. O uso de óculos de proteção e máscara visa impedir que respingos de sangue ou outros fluidos corporais, comuns na odontologia, atinjam as mucosas ocular, nasal e bucal dos profissionais. Roupas de proteção com mangas longas são indicadas para reduzir a contaminação da roupa com sangue ou outros fluidos corporais. O gorro não possui papel fundamental na prevenção das exposições ocupacionais, contudo impede a deposição de gotículas e partículas geradas durante o atendimento no cabelo do profissional, limitando a 
Tabela 5 - Análises bruta e ajustada, através de regressão de Poisson, da associação entre vacinação completa contra a hepatite B e variáveis demográficas, ocupacionais e educacionais entre cirurgiões-dentistas, Florianópolis, Brasil, 2004.

Table 5 - Crude and adjusted analysis, through Poisson regression, of the association between complete series vaccination against hepatitis B and demographic, occupational and educational variables among dentists, Florianopolis, Brazil, 2004.

\begin{tabular}{|c|c|c|c|c|c|}
\hline \multirow[b]{2}{*}{ Variáveis } & \multirow[b]{2}{*}{$\begin{array}{c}\text { Prevalência } \\
\text { da vacinação (\%) }\end{array}$} & \multicolumn{2}{|c|}{ Análise bruta } & \multicolumn{2}{|c|}{ Análise ajustada a } \\
\hline & & $\begin{array}{c}R P^{*} \\
(I C 95 \% * *)\end{array}$ & Valor $\mathrm{p}^{\S}$ & $\begin{array}{c}\mathrm{RP} * \\
(\mathrm{IC} 95 \% * *)\end{array}$ & Valor $\mathrm{p}^{\S \S}$ \\
\hline Sexo & & & 0,183 & & 0,152 \\
\hline Feminino & 77,0 & 1,0 & & 1,0 & \\
\hline Masculino & 69,5 & $0,90(0,78 ; 1,04)$ & & $0,90(0,78 ; 1,04)$ & \\
\hline Idade (anos) & & & 0,687 & & 0,558 \\
\hline$<40$ & 72,0 & 1,0 & & 1,0 & \\
\hline$\geq 40$ & 74,8 & $1,04(0,90 ; 1,19)$ & & $1,04(0,91 ; 1,19)$ & \\
\hline Ano da formatura & & & 0,060 & & 0,039 \\
\hline$<1997$ & 70,4 & 1,0 & & 1,0 & \\
\hline$\geq 1997$ & 82,6 & $1,17(1,02 ; 1,35)$ & & $1,16(1,01 ; 1,33)$ & \\
\hline Especialidade & & & 0,175 & & 0,028 \\
\hline Não-Cirúrgicas & 72,0 & 1,0 & & 1,0 & \\
\hline Cirúrgicas ${ }^{\text {b }}$ & 85,7 & $1,19(1,00 ; 1,41)$ & & $1,24(1,02 ; 1,51)$ & \\
\hline Docência & & & 0,089 & & 0,251 \\
\hline Não & 75,6 & 1,0 & & 1,0 & \\
\hline Sim & 63,6 & $0,84(0,68 ; 1,04)$ & & $0,88(0,69 ; 1,09)$ & \\
\hline Pós-graduação & & & 0,593 & & 0,576 \\
\hline Não & 71,8 & 1,0 & & 1,0 & \\
\hline Sim & 75,2 & $1,05(0,91 ; 1,20)$ & & $1,05(0,89 ; 1,23)$ & \\
\hline Curso de atualização nos últimos 2 anos & & & 0,253 & & 0,232 \\
\hline Não & 77,8 & 1,0 & & 1,0 & \\
\hline Sim & 71,1 & $0,91(0,79 ; 1,05)$ & & $0,92(0,79 ; 1,06)$ & \\
\hline Curso de biossegurança nos últimos 2 anos & & & 0,340 & & 0,258 \\
\hline Não & 71,9 & 1,0 & & 1,0 & \\
\hline Sim & 78,5 & $1,09(0,94 ; 1,27)$ & & $1,10(0,93 ; 1,31)$ & \\
\hline
\end{tabular}

${ }^{\text {a }}$ Ajustada para sexo, ano da formatura e especialidade $/{ }^{a}$ Adjusted for gender, graduation year and specialty.

${ }^{\text {b } C i r u r g i a ~ b u c o m a x i l o f a c i a l, ~ i m p l a n t o d o n t i a, ~ p e r i o d o n t i a ~ / ~}{ }^{\circ}$ Bucomaxilofacial surgery, implant dentistry, periodontology

* Razões de prevalências / * Prevalence ratio

**Intervalos de confiança de $95 \%$ / ** $95 \%$ confidence intervals

${ }^{\S}$ Teste exato de Fisher bicaudal / ${ }^{\S}$ Two-tailed Fisher's exact test

${ }^{\S \S}$ Teste de heterogeneidade / ${ }^{\S}$ Heterogeneity test

transmissão de patógenos não apenas para o profissional, mas também para seus familiares e outros pacientes.

No presente estudo, a maioria dos CDs relatou sempre usar luvas $(91,0 \%)$, máscara $(93,8 \%)$, jaleco $(92,7 \%)$ e óculos de proteção $(59,5 \%)$. O uso de óculos de proteção entre CDs pode ser considerado baixo, já que ele é um EPI fundamental, tendo em vista que foi documentada a transmissão do HBV através de respingo de sangue à mucosa ocular ${ }^{3}$.

O EPI mais usado pelos ACDs é o jaleco $(91,3 \%)$. Esse fato pode ser atribuído ao jaleco ser usado como uniforme pelos ACDs. Considerando apenas o uso do jaleco de mangas longas, que é o tipo indicado como equipamento de proteção, a prevalência de uso foi bem menor $(48,1 \%$ entre os CDs e $40,4 \%$ entre os ACDs). Infelizmente, parece que seu uso está mais vinculado a uma questão estética do que à consciência da necessidade de proteção pessoal.

$\mathrm{O}$ uso de gorro foi considerado baixo entre CDs (28,0\%) e ACDs (26,0\%). Devido a esse EPI não ser fundamental para prevenção das exposições ocupacionais, a 
baixa prevalência de seu uso pode indicar pouca preocupação dos CDs e ACDs em evitar a contaminação cruzada, ou seja, o transporte de microorganismos de um paciente para outro.

A vacinação contra a hepatite B é um importante adjunto às precauções padrão para profissionais da saúde que podem sofrer exposições ocupacionais a sangue e fluidos corporais ${ }^{3}$. A primeira vacina contra a hepatite B foi disponibilizada em $1982^{4}$.

No Brasil, foi apenas a partir de 1995 que ela passou a ser administrada gratuitamente para profissionais e estudantes de odontologia. Antes disso, Jorge et al. (1996) ${ }^{6}$ pesquisaram 740 CDs brasileiros em um congresso da área em 1994 e constataram que $34,7 \%$ haviam sido vacinados contra a hepatite B. Apesar dessa proporção ser baixa, ela foi considerada um grande avanço, já que em um estudo similar realizado em 1990 foi encontrada uma proporção de $9,3 \%^{7}$.

Em outro estudo brasileiro, Medeiros et al. (1998) ${ }^{8}$ verificaram que $89,4 \%$ dos alunos do último período do curso de odontologia de seis faculdades do Rio de Janeiro tinham sido vacinados contra a hepatite $\mathrm{B}$; contudo, apenas $4 \%$ tinham realizado o teste para verificação da imunidade e $53,6 \%$ não sabiam da necessidade da realização desse teste.

Alves-Rezende e Lorenzato (2000) ${ }^{9}$ aplicaram um questionário a $80 \mathrm{CDs}$ da região centro-oeste do Estado de São Paulo e observaram que quase todos os 69 respondentes informaram ter tomado as três doses da vacina contra a hepatite B $(90,6 \%)$. Todavia, quase metade dos CDs $(44,2 \%)$ empregava ACDs não vacinados contra a hepatite B.

Martins e Barreto (2003) ${ }^{10}$ determinaram a prevalência e os fatores associados à vacinação contra a hepatite B entre 299 CDs residentes em Montes Claros, Estado de Minas Gerais. Dentre os 296 CDs que responderam ao questionário, $74,9 \%$ haviam tomado as três doses da vacina, indicando que aproximadamente um quarto dos profissionais não realizaram o esquema vacinal completo e, entre estes últimos, $10 \%$ não haviam recebido nenhuma dose da vacina. A principal razão alegada para a não vacinação ou vacinação incompleta foi a falta de informação. Alguns dos fatores associados à não vacinação foram: idade acima de 40 anos, formatura há pelo menos 15 anos, não realização de cursos de reciclagem nos últimos dois anos e gênero masculino.

A prevalência da vacinação completa contra a hepatite B entre CDs no presente estudo $(73,4 \%)$ foi bastante similar àquela reportada por Martins e Barreto (2003) ${ }^{10}$. Contudo, não foi investigado se os sujeitos vacinados com menos de três doses estavam no intervalo entre doses e viriam a completar o esquema vacinal. Dessa forma, a prevalência da vacinação completa pode ter sido subestimada no presente estudo.

Comparando com estudos estrangeiros, a prevalência da vacinação completa entre CDs no presente estudo foi semelhante àquela observada na Alemanha $(74,0 \%)^{11}$ e na Tailândia $(68,0 \%)^{12}$, mas ficou abaixo daquela observada na África do Sul $(90,0 \%)^{13}$ e no Reino Unido $(98,9 \%$, $99,0 \%)^{14,15}$.

Considerando que a primeira campanha de vacinação contra a hepatite B para profissionais e estudantes da odontologia no Brasil foi implementada em 1995, para a análise dos dados do presente estudo deu-se um intervalo de dois anos para que começassem a se formar os profissionais vacinados durante a graduação. Observouse que o ano de formatura a partir de 1997 foi um fator associado à vacinação completa, após o ajuste para sexo e especialidade, na análise multivariável. Esse achado pode sugerir que a vacinação, ainda durante o curso de graduação, além de conferir a imunização antes do contato com pacientes, também garante uma maior cobertura vacinal na população de CDs, já que, na universidade, os estudantes recebem incentivo à vacinação e têm facilidade de acesso à vacina, que é adminis- 
trada em seu local de estudo, enquanto os cirurgiões-dentistas formados antes da campanha necessitam procurar uma unidade de saúde munidos de documentação para receber a vacina.

No presente estudo, além do ano de formatura a partir de 1997, a atuação predominante em especialidade cirúrgica - o que evidentemente predispõe a mais exposições ocupacionais a sangue - também foi positivamente associada a uma maior prevalência da vacinação completa contra a hepatite B entre CDs na análise multiváriável, fato que não foi observado na análise bruta. Dessa forma, acredita-se que o sexo seja um fator de confusão nessa associação. A prevalência da vacinação completa contra a hepatite $B$ foi mais elevada entre CDs mulheres $(77,0 \%)$ do que entre homens $(69,5 \%)$, apesar de não haver significância estatística na associação (Tabela 5). Acredita-se que essa diferença entre os gêneros reflita uma maior consciência preventiva, ou um maior cuidado em relação à proteção pessoal contra os riscos biológicos, em especial contra a hepatite $\mathrm{B}$, entre as mulheres.

Surpreendentemente, a atuação na docência não foi associada a maior prevalência de vacinação completa contra a hepatite B; pelo contrário, essa prevalência foi maior entre os CDs que não realizavam atividade de docência em odontologia $(75,6 \%)$ do que entre aqueles que a realizavam $(63,6 \%)$, apesar de não haver significância estatística na associação (Tabela 2). A condição de docente deveria estar fortemente associada à vacinação completa, já que eles são modelos de identificação para os estudantes, futuros CDs.

Outro achado do presente estudo foi que a realização de curso de atualização ou aperfeiçoamento e de curso envolvendo biossegurança nos últimos dois anos não foi associada a um índice mais elevado de vacinação contra a hepatite B. Inclusive a prevalência de vacinação completa entre os CDs que haviam realizado curso de atualização nos últimos dois anos $(71,1 \%)$ foi menor do que entre aqueles que não a haviam realizado $(77,8 \%)$. Esse fato sugere que esses cursos não estão abordando medidas de proteção individual contra a infecção. Frente a isso, sugere-se a inclusão de conteúdos de biossegurança não apenas nos cursos de pós-graduação em odontologia, mas também nos cursos de atualização ou aperfeiçoamento, de modo a estimular a vacinação dos profissionais já formados e não vacinados, ou com vacinação incompleta.

O presente estudo também avaliou a vacinação contra a hepatite B entre ACDs e verificou-se que apenas 39,4\% dos ACDs haviam completado o esquema vacinal. Da mesma maneira que com os CDs, essa prevalência pode ter sido subestimada. Entretanto, outros estudos também verificaram que a prevalência da vacinação completa contra a hepatite $\mathrm{B}$ foi mais baixa entre ACDs do que CDs ${ }^{11,13,14}$. As diferenças nos achados entre CDs e ACDs quanto às medidas de proteção pessoal contra a hepatite $\mathrm{B}$ podem estar relacionadas não apenas ao menor conhecimento na categoria dos ACDs, mas também a aspectos como a hierarquia e a subordinação dos ACDs aos CDs, em função da divisão social e técnica do trabalho, bem como da relação de emprego dos primeiros em relação aos últimos.

Moore et al. (2003) ${ }^{15}$ examinaram a disponibilidade de programas de vacinação e monitoramento sorológico para profissionais da odontologia na Escócia. Dentre 194 CDs pesquisados, 99,0\% haviam realizado vacinação e, destes, 99,0\% haviam completado o esquema vacinal. Dentre os 279 ACDs pesquisados a aderência foi mais baixa: $97,1 \%$ haviam sido vacinados e $94,8 \%$ haviam realizado o esquema vacinal completo. A verificação da imunização pós-vacinação variou de $87 \%$ a $98 \%$ em diferentes regiões da Escócia.

No presente estudo, a verificação da imunização foi considerada baixa. Apenas $32,1 \%$ dos CDs e $21,9 \%$ dos ACDs que completaram o esquema vacinal realizaram exames.

É importante que os profissionais da 
saúde realizem exame de sangue para verificar o título de anticorpos anti-HBS para verificar a imunidade contra a hepatite $B$ até um mês após a última dose do esquema vacinal. Os profissionais que não desenvolvem um nível de anticorpos adequado (anti-HBs > 10mUI/mL) após o esquema vacinal primário devem completar uma segunda série de três doses da vacina ou ser avaliados para determinar se são portadores do $\mathrm{HBV}^{3}$. Os indivíduos nãorespondedores devem ser alertados de que são suscetíveis ao HBV e a receber imunoglobulina contra a hepatite B caso sofram exposição ao HBV. Além disso, com o passar do tempo, os títulos de anticorpos no sangue declinam, porém os respondedores à vacina que perdem o anti-HBs detectável permanecem protegidos contra a infecção pelo HBV, podendo ser equivocadamente classificados como não-respondedores ${ }^{3}$. Isso reforça a importância da realização da verificação da imunidade um mês após a última dose do esquema vacinal, o que pode evitar a realização desnecessária da revacinação.

A situação apontada no presente estudo, com tantos profissionais não tendo sido vacinados e grande parte dos vacinados não conhecendo seu estado sorológico frente à hepatite $\mathrm{B}$, é preocupante. Fica evidente que são necessárias medidas educativas voltadas para CDs e principalmente para os ACDs, visando a vacinação daqueles que não o fizeram ou não completaram o esquema vacinal e a verificação da imunidade naqueles que realizaram o esquema vacinal completo. Além disso, recomenda-se a continuidade da vacinação dos estudantes de odontologia nas universidades, porém informando sobre a necessidade do monitoramento da resposta vacinal.
Para a avaliação de programas, poderão ser realizados estudos transversais do tipo painel, comparando-os com as observações deste estudo. Devido à restrição da amostra ao município de Florianópolis, é necessário o estudo de uma população maior, com amostragem probabilística, para a avaliar a aderência às medidas de proteção individual contra a hepatite $B$ entre os CDs e ACDs brasileiros.

A vacinação, juntamente com a aderência às medidas de precaução padrão, consciência e cuidado por parte dos profissionais, é fundamental para evitar a transmissão do HBV na prática odontológica, visando proteger não apenas os profissionais, mas também seus familiares e pacientes.

\section{Colaboradores}

L. P. Garcia e V. L. G. Blank contribuíram na concepção e desenho do estudo. L. P. Garcia realizou a coleta dos dados, a organização do banco de dados, a análise dos dados e redigiu a base do artigo. $\mathrm{N}$. Blank contribuiu com a análise estatística. V. L. G. Blank orientou todas as etapas e participou da elaboração final do manuscrito.

\section{Agradecimentos}

Este trabalho é parte da dissertação de Mestrado em Saúde Pública da primeira autora, apresentada ao Programa de PósGraduação em Saúde Pública da Universidade Federal de Santa Catarina, ao qual os autores agradecem o apoio. A primeira autora agradece à CAPES pela concessão de bolsa. Os autores agradecem a todos os cirurgiões-dentistas e auxiliares de consultório dentário que participaram deste estudo.

\section{Referências}

1. Brasil. Ministério da Saúde. Secretaria de Vigilância em Saúde. Departamento de Análise de Situação de Saúde. Situação da prevenção e controle das doenças transmissíveis no Brasil. In: Ministério da Saúde. Saúde Brasil 2004: uma análise da situação de saúde. Brasília:
Ministério da Saúde; 2004.

2. Brasil. Ministério da Saúde. Controle de Infecções e a prática odontológica em tempos de AIDS: manual de condutas. Brasília; 2000. 
3. Kohn WG, Collins AS, Cleveland JL, Harte JA, Eklund KJ, Malvitz DM; Centers for Disease Control and Prevention (CDC). Guidelines for infection control in dental healthcare settings - 2003. MMWR Recomm Rep 2003; 52: 1-66.

4. Cleveland JL, et al. Factors associated with hepatitis B vaccine response among dentists. J Dent Res 1994; 73: 1029-35.

5. Garcia LP, Blank VLG. Prevalência de exposições ocupacionais de cirurgiões-dentistas e auxiliares de consultório dentário a material biológico. Cad Saúde Pública 2006; 22: 97-108.

6. Jorge J, Jorge R, De Almeida OP, Scully C. Knowledge of and attitudes about blood-borne viruses and infection control in Brazilian dental practice. Oral Dis 1996; 2: 41-4.

7. de Almeida OP, Scully C, Jorges J. Hepatitis B vaccination and infection control in Brazilian dental practice, 1990. Community Dent Oral Epidemiol 1991; 19: 225-7.

8. Medeiros UV, Cardoso AS, Ferreira SMS. Uso das normas de controle de infecção na prática odontológica. Rev Bras Odontol 1998; 55: 209-15.

9. Alves-Rezende MCR, Lorenzato F. Avaliação dos procedimentos de prevenção dos riscos biológicos por cirurgiões-dentistas. Rev Assoc Paul Cir Dent 2000; 54: 446-54.
10. Martins AMEBL, Barreto, SM. Vacinação contra a hepatite B entre cirurgiões dentistas. Rev Saúde Pública 2003; 37: 333-8.

11. Ammon A, Reichart PA, Pauli G, Petersen LR. Hepatitis B and $\mathrm{C}$ among Berlin dental personnel: incidence, risk factors, and effectiveness of barrier prevention measures. Epidemiol Infect 2000; 125: 407-13.

12. Leggat PA. et al. Occupational hygiene practices of dentists in southern Thailand. Int Dent J2001; 51: 11-6.

13. Yengopal V, Naidoo S, Chikte UM. Infection control among dentists in private practice in Durban. SADJ2001; 56: 580-4.

14. Whittle JG. An audit of the hepatitis B vaccination status of staff in general dental practices in Lancashire. Prim Dent Care 2003; 10:27-9.

15. Moore MA, Macpherson LM, Kennedy C, Bagg J. Provision of hepatitis B vaccination for primary care dental staff in Scotland. J Infect 2003; 47: 322-7.

Recebido em: 28/12/07

Versão final reapresentada em: 06/08/07

Aprovado em: 27/08/07 